\title{
Síndrome de la articulación temporomandibular en un área de salud
}

\section{Temporomandibular joint syndrome in a health area}

C. Martín Marín*, D. Vega García**, R. Ramos Pastor***, A. Gallardo Ponce****, C. Navarro López $* * * *$, M. Andrés Mateo******

\section{RESUMEN}

Introducción: La alteración de la articulación temporomandibular es un motivo de consulta cada vez más común. Para caracterizar este proceso, se estudiaron pacientes diagnosticados en cuatro consultas de odontología de atención primaria durante los años 2014 a 2106.

Material y Métodos: estudio descriptivo transversal de todos los pacientes que acudieron a consulta, a los que se les diagnosticó esta patología.

Resultados: Se estudiaron 228 pacientes, lo que supone un 2.9\% de todas las consultas, habiéndose incrementado. Estos pacientes tenían una edad entre 13 y 88 años. El $75.8 \%$ de ellos presentaban dos o más factores de riesgo. Los factores de riesgo más frecuentes fueron la pérdida de dientes (56.8\%), el estrés (46.9\%) y las inferencias oclusales (43.8\%). Sin embargo estos factores varían en función de la edad y del género; así la pérdida de dientes está presente en el $83.9 \%$ de las personas de 65 a 74 años, mientras que el estrés es más frecuente en el grupo de edad de 35 a 44 años. Por otro lado, las mujeres tienen más frecuentemente estrés y bruxismo que los hombres.

Discusión: La prevalencia encontrada es algo inferior a la descrita en la literatura, sin embargo se encuentra una tendencia creciente. Los factores de riesgo de nuestros pacientes muestran diferencias en cuanto a la edad y al género.

PALABRAS CLAVE: Trastornos de la Articulación Temporomandibular; prevalencia; factores de riesgo.

\begin{abstract}
Introduction: The alteration of the temporomandibular joint is an increasingly common reason for consultation. To characterize this process, patients diagnosed in four primary care dentistry consultations during the years 2014 to 2106 were studied.

Material and Methods: cross-sectional descriptive study of the patients who went to the clinic, who were diagnosed with this pathology.

Results: 228 patients were studied, which means $2.9 \%$ of all consultations, having increased. These patients were between 13 and 88 years old. $75.8 \%$ of them had two or more risk factors. The most frequent risk factors were tooth loss $(56.8 \%)$, stress $(46.9 \%)$ and occlusal inferences $(43.8 \%)$. However, these factors vary according to age and gender; Thus, tooth loss is present in $83.9 \%$ of people aged 65 to 74 , while stress is more frequent in the age group of 35 to 44 years. On the other hand, women have more frequently stress and bruxism than men.

* Técnico de Salud. Gerencia de Atención Primaria de Palencia (España).

** Odontólogo. Unidad de Salud Bucodental del Centro de Salud La Puebla de Palencia (España).

*** Médico-Estomatólogo. Unidad de Salud Bucodental del Centro de Salud Pintor Oliva de Palencia (España).

**** Médico-Odontólogo. Unidad de Salud Bucodental de la Zona Norte. Palencia (España).

***** Médico-Estomatólogo. Unidad de Salud Bucodental del Centro de Salud de Eras del Bosque.

Palencia (España).

****** Médico-Odontólogo de la Unidad de Salud Bucodental del Centro de Salud de Jardinillos. Palencia (España).
\end{abstract}


Discussion: The prevalence found is somewhat lower than that described in the literature; however, there is a growing trend. The risk factors of our patients show differences in terms of age and gender.

KEY WORDS: Temporomandibular joint disorders, Prevalence, Risk factor's.

Fecha de recepción: 10 de diciembre de 2019

Fecha de aceptación: 10 de febrero de 2020

C. Martín Marín, D. Vega García, R. Ramos Pastor, A. Gallardo Ponce, C. Navarro López, M. Andrés Mateo. Síndrome de la articulación temporomandibular en un área de salud. 2021; 37, (2): 94-100.

\section{INTRODUCCIÓN}

En la cartera de servicios comunes del Sistema Nacional de Salud se incluye en el nivel de Atención Primaria y para toda la población, el tratamiento de los procesos inflamatorios e infecciosos que afectan al área bucodental, traumatismos osteodentarios, heridas y lesiones en la mucosa oral, así como la patología aguda de la articulación témporo-mandibular. Incluye consejo bucodental, tratamiento farmacológico de la patología bucal que lo requiera, exodoncias, exodoncias quirúrgicas, cirugía menor de la cavidad oral, revisión oral para la detección precoz de lesiones premalignas y, en su caso, biopsia de lesiones mucosas ${ }^{(1)}$.

Las principales enfermedades que se tratan en nuestro entorno son la caries dental, la enfermedad periodontal y la maloclusión, por lo que en la población adulta se realizan habitualmente revisiones, extracciones dentales y biopsias. En ocasiones se realizan ciertos procesos quirúrgicos como regularización ósea del alveolo, drenajes o incluso exodoncias quirúrgicas de dientes incluidos y cordales, salvo que estén en íntimo contacto con el nervio, en cuyo caso se enviaría al cirujano maxilofacial.

Tradicionalmente el motivo de consulta más frecuente en la población adulta consistía en la necesidad de realizar una exodoncia, bien debido a caries o erupción de los cordales (en pacientes menores de 35 años), o por enfermedad periodontal a partir de dicha edad, ésta potenciada en gran medida por el tabaco y enfermedades o tratamientos que disminuyen las defensas (diabetes, pacientes trasplantados, deficiencias adquiridas, etc.). ${ }^{(2)}$

Sin embargo, en los últimos años parece que se está produciendo un cierto cambio de tendencia: las consultas por exodoncias van disminuyendo paulatinamente, probablemente porque la población cuida mejor la boca, y muchos de los nuevos adultos han estado cubiertos por el programa infantil ${ }^{(2)}$. Sin embargo, llama la atención que a la vez que la población adulta ha mejorado considerablemente su salud dental de forma tal que se realizan menos exodoncias y se reponen con más rapidez y frecuencia las piezas ausentes, aumentan los trastornos temporomandibulares. ${ }^{(3)}$

La alteración de la articulación temporomandibular (o síndrome de disfunción temporomandibular) se define como un conjunto de síntomas que incluye dolor, debilidad muscular, clics articulares y limitación o alteración del movimiento mandibular y que está relacionado con la articulación temporomandibular, los músculos masticatorios y la oclusión dental. Después del dolor odontogénico, los trastornos temporomandibulares (ATM) son una de las causas más comunes de dolor en la boca y la cara y también tienen el potencial de producir dolor persistente (crónico). ${ }^{(4)}$

La etiología se este trastorno se considera actualmente multifactorial y se relaciona con factores predisponentes o generales como la edad y el género, enfermedades sistémicas, psicológicas y psicosociales, y factores de riesgo locales como el bruxismo, sobrecargas mecánicas y traumatismos directos e indirectos que afecten a la articulación temporomandibular. ${ }^{(5,6,7,8,9,10)}$

Dada la importancia y frecuencia creciente de este problema, así como el hecho de que un mejor conocimiento de los factores etiológicos o desencadenantes permitirá un mejor manejo de este síndrome, se realizó este estudio para conocer la frecuencia en nuestro entorno y su relación con el sexo, la edad y otros factores. Se realizó un estudio descriptivo retrospectivo de pacientes de las unidades de salud bucodental del area de Palencia, entre los años 2014 a 2016, tratando de identificar los factores predisponentes y potenciadores que justificaran esta tendencia al alza en el número de consultas y proporcionasen estrategias de tratamiento más enfocadas y efectivas. 


\section{MATERIAL Y MÉTODOS}

Se recogió información de todos los pacientes atendidos y derivados a atención especializada en cuatro de las cinco consultas de odontología de las unidades de salud bucodental (USBD) del área de salud de Palencia, durante los años 2014 a 2016. Estas unidades, ubicadas en los centros de salud urbanos, atienden a personas que viven tanto en la zona urbana como en zonas rurales.

El diagnóstico de ATM se realizó mediante criterios clínicos (historia clínica y examen físico) y ortopantomografía en todos los casos. En un 20'9\% de los casos de realizó también resonancia magnética nuclear.

La recogida de datos se realizó por los propios odontólogos mediante la revisión de sus archivos clínicos y aplicación informática. Se rellenó un cuestionario diseñado a tal efecto, donde se incluían datos demográficos del paciente, factores de riesgo fundamentalmente de tipo mecánico, prueba diagnóstica, derivado o no, tratamiento en la Unidad (Anexo 1)

Los factores de riesgo se obtuvieron mediante una revisión de la literatura, recogiendo principalmente factores de tipo odontológico (hábitos parafuncionales, maloclusión, traumatismos, pérdidas de dientes) pero también estrés o enfermedades reumatológicas.

Se calculó la media e intervalos de confianza para las variables cuantitativas y porcentajes para las cualitativas para describir la muestra. Posteriormente se realizó un análisis bivariante de las variables cualitativas mediante tablas de 2X2, comparando la proporción de los diversos factores de riesgo en función del género (hombre vs mujer) y en función de la edad categorizada en dos grupos (menores o iguales de 30 años y mayores de 30 años), calculando la Chi cuadrado o, en su caso, la prueba exacta de Fisher.

Se estableció un nivel de confianza del 95\%.

El software utilizado para la entrada de datos y la recuperación de cálculos estadísticos fue SPSS (Paquete estadístico para las ciencias sociales - versión 19.0).

No se consideró necesario solicitar de forma expresa consentimiento informado de cada paciente, al recogerse los datos por los propios facultativos que habían tratado a cada paciente, consistir en información anonimizada que no permite en absoluto su reconocimiento expreso y tratarse de un estudio con el fin de

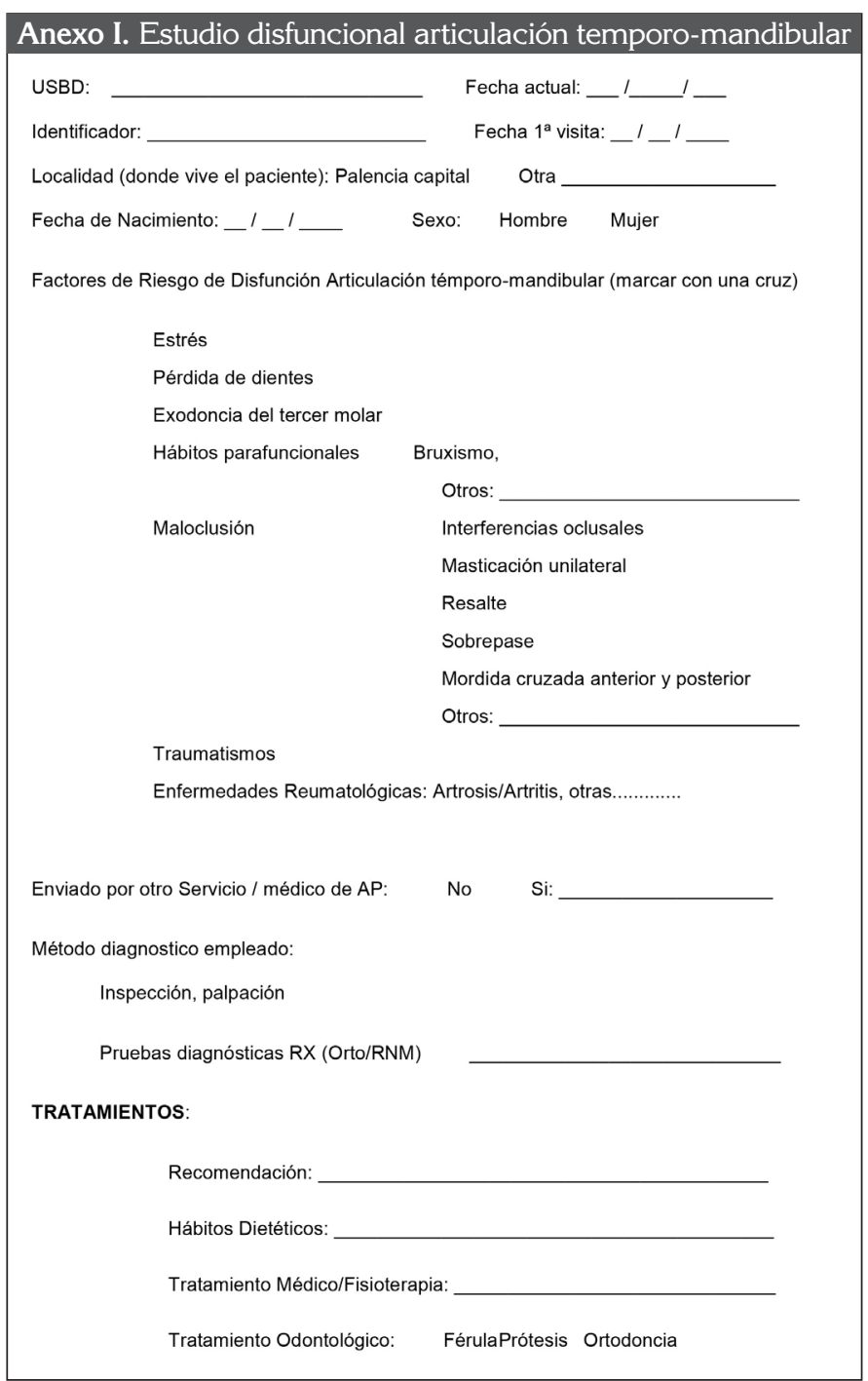

mejorar la calidad de la asistencia en el propio sistema sanitario (investigación con fines de planificación y control de calidad).

\section{RESULTADOS}

El número de pacientes atendidos en las consultas de odontología de atención primaria durante los años 2014 a 2016, por alteración en la articulación temporomandibular fue 228. Estos pacientes estaban comprendidos entre los 13 y los 88 años, con una edad media de $44^{\prime} 4$ años (IC95\%: 42’0-46'9). De ellos, 181 (79'4\%) eran mujeres, y el resto hombres (47 pacientes).

El número de pacientes por ATM atendidos en los tres años estudiados supuso un 2,92 \%0 de todos los pacientes, habiendo aumentado desde el año 2014 (2,22 


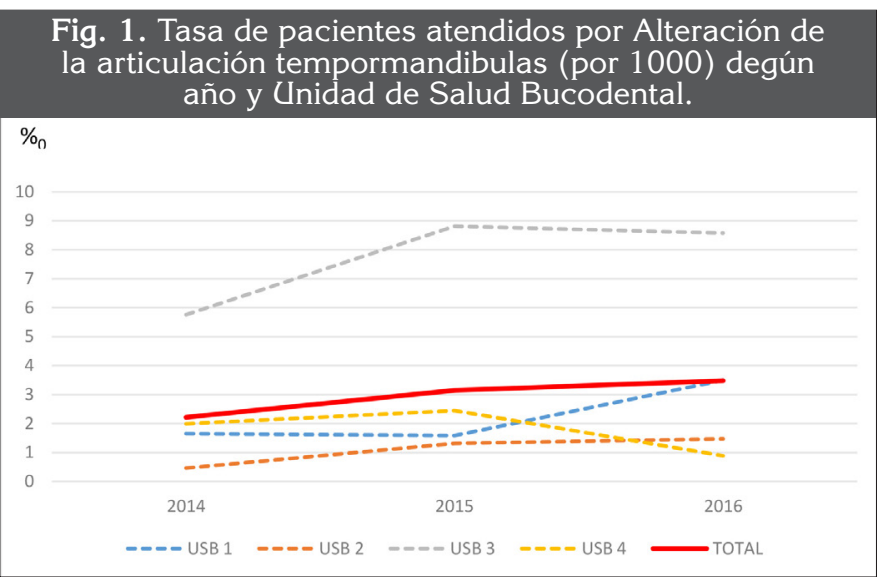

Fig. 2. Porcentaje de casos atendidos según grupo de edad $\%$
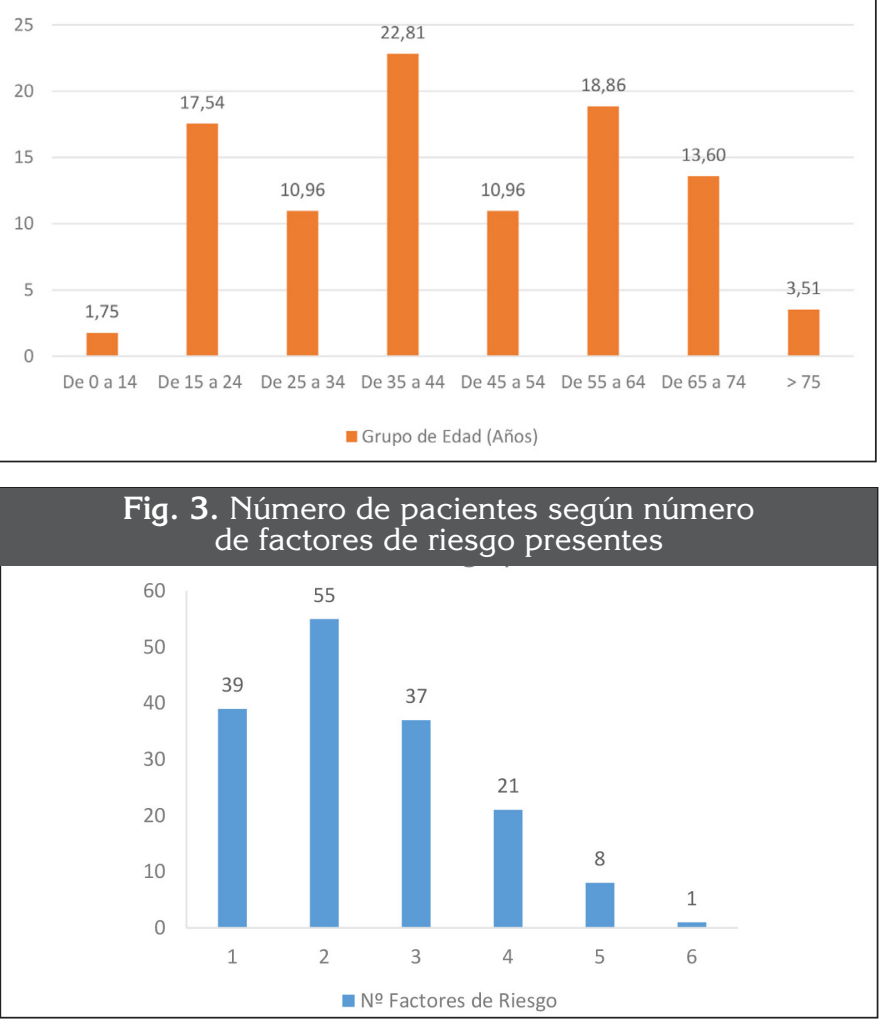

$\% 0)$ al $2016(3,48 \% 0)$. Este aumento se produjo a expensas sobre todo de la USB1 y USB3 (fig. 1).

La distribución por grupos de edad se muestra en la figura 2, donde se puede ver que, aunque la máxima incidencia de casos atendidos en las consultas se corresponde con el grupo de edad de 35 a 44 años $(22,8 \%)$, existen también dos importantes picos de incidencia en los grupos de 15 a 24 años $(17,5 \%)$ y de 55 a 64 años (18,9\%).
El lugar de residencia fue "otra localidad distinta de la capital" en 76 casos (33'3\%), mientras que el resto vivía en Palencia capital.

Se recogieron los factores de riesgo relacionados con la alteración de la articulación temporomandibular en 161 pacientes. La mayoría de los pacientes (75’8\%), presentaban dos o más factores de riesgo, mientras que solo 39 pacientes (24'2\%) referían un solo factor. (fig. 3 )

Entre los factores de riesgo, los más frecuentes fueron la pérdida de dientes $\left(56^{\prime} 8 \%\right)$, el estrés $\left(46^{\prime} 9 \%\right)$ y las interferencias oclusales (43’8\%) (fig. 4)

Por grupos de edad, la frecuencia de factores de riesgo se muestra en la Tabla 1, donde se observan las diferencias existentes según el grupo: así la pérdida de dientes está presente en el $83^{\prime} 87 \%$ de las personas de 65 a 74 años y las enfermedades reumatológicas en el $62,5 \%$ de los pacientes mayores de 75 años, mientras que el estrés es más frecuente en el grupo de edad de 35 años a 44 años. El Bruxismo es frecuente a partir de 35 años, mientras que las inferencias oclusales y otras maloclusiones están presentes muy frecuentemente en todos los grupos de edad, sobre todo en el grupo de 45 a 54 años. En los más jóvenes (de 1 a 14 años) son factores relacionados con problemas en las piezas dentarias (maloclusiones o traumatismos)

Si desagregamos estos factores de riesgo según el grupo de edad, observamos que el porcentaje varía, así los factores presentes en el grupo de edad de 1 a 14 años están relacionados con problemas en las piezas dentarias (maloclusiones o traumatismos) y en el grupo de 15 a 20 años también son más frecuentes las maloclusiones (interferencias oclusales u otras) al igual que en el grupo de 21 a 35 años. En los dos grupos de edad restantes (de 36 a 65 años y mayores de 65), el mayor porcentaje de pacientes presentan pérdida de dientes y estrés, aunque también maloclusiones. En el grupo de mayores de 65 años tienen un importante peso las enfermedades reumatológicas, presentes en el $50 \%$ de los pacientes.

En el análisis bivariante se encontraron diferencias estadísticamente significativas en relación con el género (hombre-mujer) para la prevalencia de factores de riesgo de estrés y bruxismo, mientras que no se encontraron diferencias en el resto de factores estudiados (pérdida de dientes, interferencias oclusales, otra maloclusión y enfermedades reumatológicas. En las siguientes tablas se muestran los porcentajes en los que se encontraron diferencias estadísticamente significa- 


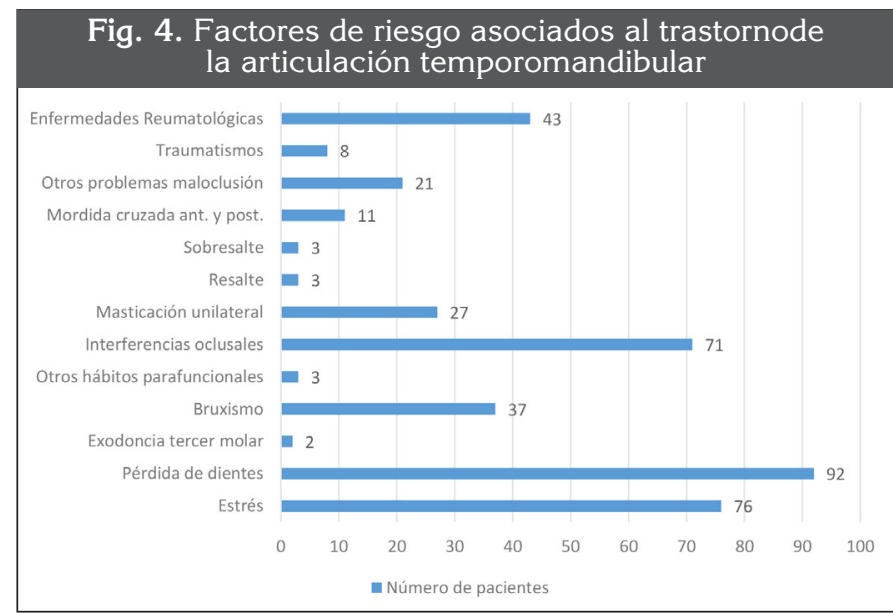

\begin{tabular}{|c|c|c|c|c|c|c|c|c|c|c|c|c|c|c|c|c|c|c|}
\hline \multirow{2}{*}{$\begin{array}{c}\text { Grupo de Edad } \\
\text { (Años) / Factores } \\
\text { de Riesgo }\end{array}$} & \multicolumn{2}{|c|}{1 a 14} & \multicolumn{2}{|c|}{15 a 24} & \multicolumn{2}{|c|}{25 a 34} & \multicolumn{2}{|c|}{35 a 44} & \multicolumn{2}{|c|}{45 a 54} & \multicolumn{2}{|c|}{$\begin{array}{l}55 a \\
64 \\
\end{array}$} & \multicolumn{2}{|c|}{65 a 74} & \multicolumn{2}{|c|}{$>75$} & \multicolumn{2}{|c|}{\begin{tabular}{|c} 
TODOS LOS \\
GRUPOS \\
EDAD \\
\end{tabular}} \\
\hline & $\mathbf{N}$ & $\%$ & $\mathrm{~N}$ & $\%$ & $\mathbf{N}$ & $\%$ & $\mathbf{N}$ & $\%$ & $\mathbf{N}$ & $\%$ & $\mathrm{~N}$ & $\%$ & $\mathbf{N}$ & $\%$ & $\mathbf{N}$ & & \begin{tabular}{|l|l|}
$\mathbf{N}$ & \\
\end{tabular} & $\%$ \\
\hline Estrés & 0 & 0 & 9 & 20 & 9 & 41 & 22 & 22 & 9 & 21 & 15 & 18 & 9 & 11 & 3 & 17 & 76 & 19,1 \\
\hline Pérdida de Dientes & 0 & 0 & 4 & 9,2 & 3 & 14 & 23 & 23 & 7 & 16 & 25 & 29 & 26 & 33 & 4 & 22 & 92 & 23,2 \\
\hline Exod. 3er molar & 0 & 0 & 1 & 2,3 & 0 & 0 & 1 & 1,1 & 0 & 0 & 0 & 0 & 0 & 0 & 0 & 0 & 2 & 0,5 \\
\hline Bruxismo & 0 & 0 & 5 & 11 & 2 & 9,1 & 11 & 11 & 5 & 12 & 6 & 7,1 & 7 & 8,8 & 1 & 5,6 & 37 & 9,3 \\
\hline $\begin{array}{l}\text { Otros hábitos } \\
\text { disfuncionales }\end{array}$ & 0 & 0 & 0 & 0 & 0 & 0 & 1 & 1,1 & 0 & 0 & 1 & 1,2 & 1 & 1,2 & 0 & 0 & 3 & 0,8 \\
\hline $\begin{array}{l}\text { Interferencias } \\
\text { oclusales }\end{array}$ & 2 & 33 & 5 & 11 & 4 & 18 & 20 & 20 & 10 & 23 & 15 & 18 & 12 & 15 & 3 & 17 & 71 & 17,9 \\
\hline $\begin{array}{l}\text { Masticación } \\
\text { unilateral }\end{array}$ & 0 & 0 & 2 & 4,5 & 1 & 4,5 & 4 & 4 & 5 & 12 & 7 & 8,3 & 8 & 10 & 0 & 0 & 27 & 6,8 \\
\hline $\begin{array}{l}\text { Resalte I } \\
\text { Sobrepase }\end{array}$ & 1 & 16,7 & 2 & 4,5 & 0 & 0 & 0 & 0 & 2 & 4,6 & 1 & 1,2 & 0 & 0 & 0 & 0 & 6 & 1,5 \\
\hline Mordida cruzada & 0 & 0 & 3 & 6,8 & 2 & 9,1 & 3 & 3 & 0 & 0 & 2 & 2,3 & 0 & 0 & 1 & 5,6 & 11 & 2,8 \\
\hline $\begin{array}{l}\text { Otras } \\
\text { Maloclusiones }\end{array}$ & 1 & 17 & 6 & 14 & 1 & 4,5 & 4 & 4 & 2 & 4,7 & 3 & 3,5 & 3 & 3,7 & 1 & 5,6 & 21 & 5,3 \\
\hline Traumatismos & 2 & 33 & 1 & 2,3 & 0 & 0 & 2 & 2 & 1 & 2,3 & 1 & 1,2 & 11 & 1,3 & 0 & 0 & 8 & 2,0 \\
\hline $\begin{array}{l}\text { Enf. } \\
\text { Reumatológicas }\end{array}$ & 0 & 0 & 6 & 14 & 0 & 0 & 8 & 8,1 & 2 & 4,6 & 9 & 11 & 13 & 16 & 5 & 28 & 43 & 10,8 \\
\hline $\begin{array}{l}\text { TOTAL } \\
\text { FACTORES DE } \\
\text { RIESGO }\end{array}$ & 6 & 100 & 44 & 100 & 22 & 100 & \begin{tabular}{l|l}
99 & 1
\end{tabular} & 100 & 43 & 100 & 85 & 100 & 80 & 100 & 18 & 100 & 397 & 100,0 \\
\hline
\end{tabular}

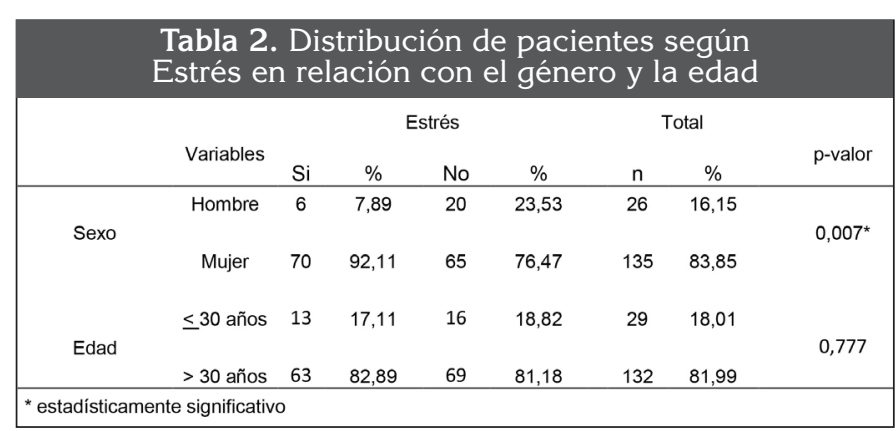

tivas según género y/o grupo de edad. (Tablas 2 a 6)

\section{DISCUSIÓN}

Este estudio como limitación, ya que es un estudio transversal, no permite evaluar relaciones de causa-efecto; sin embargo ya está sobradamente aceptado que la alteración de la articulación temporomandibular se trata de un trastorno multifactorial con factores predisponentes, desencadenantes y perpetuantes ${ }^{(12)}$.

Por otro lado, solo se describen los pacientes que han acudió a la consulta, por lo que pueden considerarse los casos con una mayor afectación de la ATM, lo que no obstante no invalida nuestros resultados.

Pretendemos con este estudio, valorar la prevalencia de los diversos factores descritos en la literatura en las personas que acuden al primer nivel de atención, es decir, a una consulta de atención primaria de salud bucodental, para lo que estudiamos todas las consultas atendidas con este diagnóstico a nivel del área de Atención Primaria de Palencia en el periodo comprendido entre los años 2014 y 2016.

En nuestro estudio, el porcentaje de casos de ATM atendidos en las consultas de odontología fue en el promedio de los tres años estudiados de un 2'92\% del total de casos vistos en consulta, lo que es ligeramente menor a la prevalencia descrita en adultos y ancianos para nuestro entorno. En el estudio de Javier Montero y cols., que encuentran una elevación de la prevalencia de dolor y disfunción de la articulación temporomandibular de 2'39 a 4'29 en $2015^{(3)}$. En Estados Unidos, la prevalencia de ATM ha sido descrita como entre el $5 \%$ y el $12 \%$ de la población ${ }^{(13)}$.

En nuestro estudio, el porcentaje de pacientes atendidos con diagnóstico de alteración de la articulación temporomandibular se incrementó con el tiempo, pasando de un 2,22\% en 2014 a un 3,48\% en 2016, lo que es concordante con el citado estudio de J. Montero et al quien realizó un estudio transversal con datos de encuestas nacionales de salud bucal de las últimas dos décadas (1993, 2000, 2005, 2010, y 2015), identificando un incremento en la prevalencia con el tiempo tanto en adultos como en ancianos ${ }^{(3)}$. Igualmente, un estudio de cohorte realizado en Suecia y que estudió la tendencia de aparición de síntomas de ATM en adultos y ancianos en un periodo de tiempo similar, también encontró un incremento significativo en la prevalencia de TMD durante este periodo ${ }^{(14)}$.

Nosotros encontramos que el $79^{\prime} 4 \%$ de los pacientes estudiados eran mujeres, lo que concuerda con la mayoría de los estudios realizados sobre ATM, que describen una prevalencia en mujeres aproximadamente 3 veces mayor que en hombres ${ }^{(6,7,8,11}$, ${ }^{15,16,17,18)}$. Sin embargo Bueno $\mathrm{CH}$ et al. ${ }^{(19)}$ realizaron una revisión y meta-análisis, encontrando el doble de riesgo entre las mujeres de desarrollar ATM que en los hombres. 


\begin{tabular}{|c|c|c|c|c|c|c|c|c|}
\hline & \multirow[b]{2}{*}{ Variables } & \multicolumn{4}{|c|}{ Pérdida de dientes } & \multicolumn{2}{|c|}{ Total } & \multirow[b]{2}{*}{$\mathrm{p}$-valor } \\
\hline & & $\mathrm{Si}$ & $\%$ & No & $\%$ & $n$ & $\%$ & \\
\hline \multirow{3}{*}{ Sexo } & Hombre & 15 & 16,3 & 11 & 15,94 & 26 & 16,15 & \multirow{3}{*}{0,951} \\
\hline & & & & & & & & \\
\hline & Mujer & 77 & 83,7 & 58 & 84,06 & 135 & 83,85 & \\
\hline \multirow{3}{*}{ Edad } & $\leq 30$ años & 5 & 5,43 & 24 & 34,78 & 29 & 18,01 & \multirow{3}{*}{$0^{*}$} \\
\hline & & & & & & & & \\
\hline & $>30$ años & 87 & 94,57 & 45 & 65,22 & 132 & 81,99 & \\
\hline
\end{tabular}

Tabla 4. Distribución de pacientes según pérdida de dientes en relación con el género y la edad

\begin{tabular}{|c|c|c|c|c|c|c|c|c|}
\hline & \multirow[b]{2}{*}{ Variables } & \multicolumn{4}{|c|}{ Pérdida de dientes } & \multicolumn{2}{|c|}{ Total } & \multirow[b]{2}{*}{$\mathrm{p}$-valor } \\
\hline & & $\mathrm{Si}$ & $\%$ & No & $\%$ & $\mathrm{n}$ & $\%$ & \\
\hline \multirow{3}{*}{ Sexo } & Hombre & 15 & 16,3 & 11 & 15,94 & 26 & 16,15 & \multirow{3}{*}{0,951} \\
\hline & & & & & & & & \\
\hline & Mujer & 77 & 83,7 & 58 & 84,06 & 135 & 83,85 & \\
\hline \multirow{3}{*}{ Edad } & $\leq 30$ años & 5 & 5,43 & 24 & 34,78 & 29 & 18,01 & \multirow{3}{*}{$0^{*}$} \\
\hline & & & & & & & & \\
\hline & $>30$ años & 87 & 94,57 & 45 & 65,22 & 132 & 81,99 & \\
\hline
\end{tabular}

Tabla 5. Distribución de pacientes según

otra maloclusión en relación con el género y la edad

\begin{tabular}{|c|c|c|c|c|c|c|c|c|}
\hline & \multirow[b]{2}{*}{ Variables } & \multicolumn{4}{|c|}{ Otra maloclusión } & \multicolumn{2}{|c|}{ Total } & \multirow[b]{2}{*}{$p$-valor } \\
\hline & & $\mathrm{Si}$ & $\%$ & No & $\%$ & $n$ & $\%$ & \\
\hline \multirow{3}{*}{ Sexo } & Hombre & 7 & 12,28 & 19 & 18,27 & 26 & 16,15 & \multirow{3}{*}{0,323} \\
\hline & & & & & & & & \\
\hline & Mujer & 50 & 87,72 & 85 & 81,73 & 135 & 83,85 & \\
\hline \multirow{3}{*}{ Edad } & $\leq 30$ años & 7 & 35,00 & 22 & 15,60 & 29 & 18,01 & \multirow{3}{*}{$0,035^{\star}$} \\
\hline & & & & & & & & \\
\hline & $>30$ años & 13 & 65,00 & 119 & 84,40 & 132 & 81,99 & \\
\hline
\end{tabular}

Tabla 6. Distribución de pacientes según

mordida cruzada en relación con el género y la edad

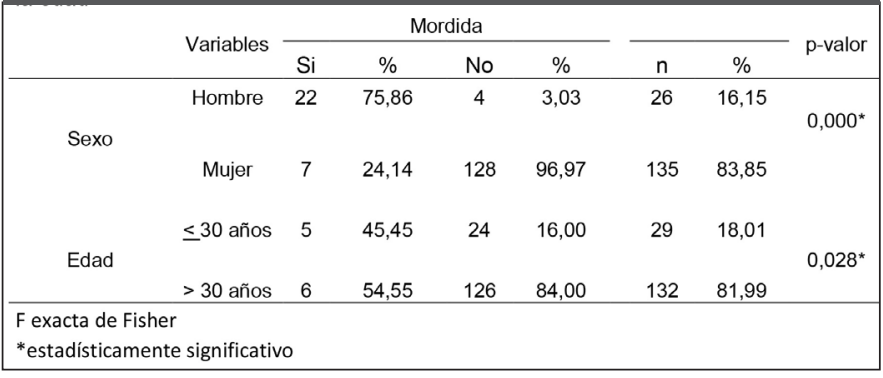

En nuestro estudio, la edad que más rango abarca es de los 25 a los 65 años (edad activa laboral y de crianza de hijos), que incluye casi el $65 \%$ de los pacientes atendidos. En otros estudios, la edad de aparición más frecuente de la ATM es entre los 20 y 40 años de edad $(6,7,8,11)$.

El 75,8\% de los pacientes atendidos por ATM presentaban dos o más factores de riesgo, lo que apoya la afirmación de que se trata de un trastorno multifactorial.
Los factores de riesgo más frecuentemente encontrados fueron la pérdida de dientes (56.8\%), el estrés (46.9\%) y las inferencias oclusales (43.8\%).

Los factores de riesgo de nuestros pacientes mostraron diferencias en cuanto a la edad; así la pérdida de dientes está presente en el 83.9\% de las personas de 65 a 74 años, mientras que el estrés es más frecuente en el grupo de edad de 35 a 44 años. En el grupo de edad de 1 a 14 años los factores de riesgo están relacionados con problemas en las piezas dentarias (maloclusiones o traumatismos), lo que concuerda con lo descrito en otras publicaciones ${ }^{(20)}$. En el grupo de 15 a 20 años también son más frecuentes las maloclusiones (interferencias oclusales u otras) al igual que en el grupo de 21 a 35 años. En los dos grupos de edad restantes (de 36 a 65 años y mayores de 65), el mayor porcentaje de pacientes presentaban pérdida de dientes y estrés, aunque también maloclusiones. En un estudio cubano que analizó las ATM en mayores de 18 años ${ }^{(21)}$ encontraron que en los pacientes de 60 a 74 la pérdida dentaria fue el factor de riesgo más frecuente; sin embargo en nuestro estudio, en el grupo de mayores de 65 años también tuvieron un importante peso las enfermedades reumatológicas, presentes en el $50 \%$ de los pacientes.

Igualmente encontramos algunas diferencias en función del género, así en mujeres es más frecuentes la existencia de estrés y bruxismo que en los hombres, mientras que en estos últimos es más frecuente la presencia de mordida cruzada que en las mujeres.

En los otros factores de riesgo (pérdida de dientes, interferencias oclusales, otras maloclusiones, enfermedades reumatológicas... no hay diferencias significativas entre los distintos sexos.

En relación con estos resultados, existen muchos estudios relativos a la mayor prevalencia de ATM en mujeres y se habla de factores sociales e incluso hormonales como explicación a este hecho, sin embargo no hemos encontrado ningún estudio como el nuestro que compare la presencia de los factores de riesgo evaluados en nuestro estudio.

Cabe entonces destacar que el modo de vida actual y las condiciones socio-laborales están llevando a un aumento de las consultas de salud bucodental por disfunción de la ATM y que el sexo femenino, con sus factores genéticos-anatómico funcionales e hiperlaxitud ligamentosa es quien está claramente destacando en 
la mayor demanda por esta patología compensando la disminución de visitas de otros pacientes por caries, periodontitis.

El odontólogo de atención primaria puede ser la pieza clave de coordinación y derivación a los diferentes profesionales implicados después de una detección precoz de problemas en la articulación temporomandibular por parte del médico de familia o la propia unidad de salud bucodental. Esta detección temprana facilitará un abordaje precoz que impida el desarrollo de una patología crónica que, en muchos casos requerirá tratamientos más agresivos en el tercer nivel (como cirugía maxilofacial).

\section{REFERENCIAS BIBLIOGRÁFICAS}

1. Cartera de Servicios del Ministerio de Sanidad. Disponible en: https://www.mscbs.gob.es/profesionales/prestacionesSanitarias/CarteraDeServicios/ContenidoCS/2AtencionPrimaria/AP-SaludBucoDental.htm. Consultado el 18 de noviembre de 2019

2. Libro Blanco: Encuesta poblacional: la salud bucodental en España 2010. Consejo de Dentistas

3. Montero J, Llodra JC, Bravo M. Prevalence of the Signs and Symptoms of Temporomandibular Disorders Among Spanish Adults and Seniors According to Five National Surveys Performed Between 1993 and 2015. J Oral Facial Pain Headache. 2018 Fall; 32 (4):349-357. doi: 10.11607/ofph.2085.

4. Schiffman E, Ohrbach R, Truelove E, Look J, Anderson G, Goulet JP. Diagnostic Criteria for Temporomandibular Disorders (DC/TMD) for Clinical and Research Applications: Recommendations of the International RDC/TMD Consortium Network and Orofacial Pain Special Interest Group. J Oral Facial PainHeadache. 28 (1): 6-27. PMID 24482784. Consultado el 18 de noviembre de 2017.

5. Oral K, BalKüçük B, Ebeo囚lu B, Dinçer S. Etiología del dolor por desorden temporomandibular. Agri 2009; 21: 89-94.

6. Bonjardim LR, Lopes-Filho RJ, Amado G, Albuquerque Junior RLC, Gonçalves SRJ. Asociación entre síntomas de trastornos temporomandibulares y género, oclusión morfológica y factores psicológicos en un grupo de estudiantes universitarios. Indian J Dent Res 2009; 20: 190-4.

7. Macfarlane TV, Kenealy P, Kingdon HA, Mohlin BO, Pilley JR, Richmond S, y otros. Estudio de cohorte de veinte años sobre la ganancia de salud del tratamiento de ortodoncia: trastornos temporomandibulares. Am J OrthodDentofacialOrthop 2009; 135: 692.e1-e8.

8. Marklund S, Wänman A. Factores de riesgo asociados con la incidencia y persistencia de signos y síntomas de trastornos temporomandibulares. Acta OdontolScand 2010; 68: 289-99.

9. Monteiro DR, Zuim PRJ, Pesqueira AA, Ribeiro PP, García AR. Relación entre la ansiedad y el dolor orofacial crónico del trastorno temporomandibular en un grupo de estudiantes universitarios. Revista de Investigación de Prostodoncia 2011; 55: 154-58.

19. Tecco S, Crincoli V, Di Bisceglie B, Saccucci M, Macrí M, Polimeni A, et al .. Signos y síntomas de trastornos de la articula- ción temporomandibular en niños y adolescentes caucásicos. The Journal of CraniomandibularPractice 2011; 29: 71-79.

11. Ingawalé $S$ EGoswami T. Articulación temporomandibular: trastornos, tratamientos y biomecánica. Annals of Biomedical Engineering 2009; 37: 976-96.

12. Internacional Headache Society: Classification and diagnostic criteria of headache disorders, cranial neuralgias and facial pain. Cephalalgia 8:suppl. 71988

13. Pain.NloDaCRF. Accesible en: http://www.nidcr.nih.gov/DataStatistics/FindDataByTopic/FacialPain. Acceso el 15 de septiembre de 2018.

14 Köhler AA, Hugoson A, Magnusson T. Prevalence of symptoms indicative of temporomandibular disorders in adults: Cross-sectional epidemiological investigations covering two decades. ActaOdontolScand 2012; 70:213-23.

15 Bueno CH, Pereira DD, Pattussi MP, Grossi PK, Grossi ML. Gender differences in temporomandibular disorders in adult populational studies: A systematic review and meta-analysis. J Oral Rehabil. 2018 Sep;45(9):720-29

16. LeResche L.: Epidemiology of temporomandibular disorders: implications for the investigation of etiologic factors. Crit Rev Oral Biol Med 1997; 8: pp. 291-05

17. Anastassaki Kohler A., Hugoson A., and Magnusson T.: Prevalence of symptoms indicative of temporomandibular disorders in adults: cross-sectional epidemiological investigations covering two decades. ActaOdontolScand 2012; 70: pp. 213-23

18. Manfredini D., Guarda-Nardini L., Winocur E., et al: Research diagnostic criteria for temporomandibular disorders: a systematic review of axis I epidemiologic findings. Oral Surg Oral Med Oral Pathol Oral RadiolEndod 2011; 112: pp. 453-62

19. Bueno CH, Pereira DD, Pattussi MP, Grossi PK, Grossi ML: Gender differences in temporomandibular disorders in adult populational studies: A systematic review and meta-analysis. J Oral Rehabil. 2018 Sep; 45(9): 720-29.

20. Seradarian PI, Perconoto C, Mascarenhas MH. Disfunciones temporomandibulares en bebés, niños y adolescentes en: Manual de referencia para Procedimientos Clínicos en Odontopediatria Latinoamericana. https://www.revistaodontopediatria. org/publicaciones/manuales/referencia-para-procedimientos-en-odontopediatria/Manual-de-Referencia-para-Procedimientos-en-Odontopediatria-Capitulo-24.pdf Acceso 20 de noviembre de 2018

21. Castro Gutiérrez I, Pérez Muro Y, Bermúdez Paredes M, Fernández Serrano JM. Trastornos de la articulación temporomandibular en la población mayor de 18 años del municipio Trinidad 2010. GacMédEspirit vol.17 no.2 Sancti Spíritus mayo.-ago. 2015

\section{DIRECCIÓN DE CORRESPONDENCIA:}

Primer autor: Cristina Martín Marín.

Paseo de Atapuerca s/n (09071-Burgos)

947-280100 (ext. 820813).

E-mail: cristina.martin.marin@jcyl.es

Cristina Martín Marín, David Vega García, Raúl Ramos

Pastor, Asunción Gallardo Ponce, Carmen Navarro

López, Manuel Andrés Mateo.

Síndrome de la articulación temporomandibular

en un area de salud.

Av Odontoestomatol 